\title{
ENTROPY ESTIMATION METHOD USING HEAT IN PISTON ENGINES WITH NON- EQUILIBRIUM OF INSIDE-CYLINDER PROCESSES
}

\author{
Rahim Zainetdinov $^{1}$, Irina Belinskaia ${ }^{2}$, Viktor Smelik ${ }^{1}$, Mihail Novikov ${ }^{1}$ \\ ${ }^{1}$ Saint-Petersburg State Agrarian University, Russia; \\ ${ }^{2}$ Leningrad State University named after Alexander Pushkin, Russia \\ zra61@mail.ru,belinska@yandex.ru, smelik_va@mail.ru,mihanov25@rambler.ru
}

\begin{abstract}
Resource-saving technologies are now actively used in the field of piston design. The modernization of internal combustion engines should be based on evaluation of the efficiency. The development of new methods for assessing the thermodynamic efficiency of heat use in piston engines is an essential part of improving the efficiency of motor vehicles. At present, when assessing the efficiency of thermodynamic processes in internal combustion engines of the indicator efficiency, the essence of the process of converting the thermal form of energy into mechanical work is not considered. In this regard, there is a need to study the working processes of piston engines to develop a more advanced method. The article proves the prospects of using the entropy approach and variational principles of nonequilibrium thermodynamics. This method is based on the first and second laws of thermodynamics, and allows taking into account not only the quantity, but also the quality of energy. The differential equation of thermal balance of the engine taking into account heat dissipation in the engine is offered. This equation assumes establishment of functional dependence of production of entropy in systems on parameters of the irreversible thermodynamic processes proceeding in it, and also search of optimum of the received objective function. The proposed method of entropy analysis of nonequilibrium thermodynamic processes in piston engine systems allows to evaluate the degree of efficiency of each system and to investigate the maximum capabilities of the piston engine. As a result of using the proposed method, heat losses in the engine systems are reduced.
\end{abstract}

Keywords: indicator efficiency coefficient, entropy production, heat balance, irreversible process, amount of heat.

\section{Introduction}

At a time when the world economy is entering the resource - and energy-saving way of development, the development of new methods for assessing the thermodynamic efficiency of heat use in piston engines is extremely important. This makes it possible to assess the level of technical improvement in the field of engine building and the expected prospects for modernization of internal combustion engines.

It is known that the generally accepted indicator of energy efficiency of thermodynamic processes in internal combustion engines is the indicator efficiency. In the development of the research methods of indicated efficiency, a great contribution was made by Russian and foreign scientists: V. Grinevskiy, N. Breling, a. Vanshteydt, N. Glagolev, K. Genkin, N. Dyachenko, A. Kudruash, T. Melkumov, D. Matvievskiy, D. Portnov, S. Pogodin, V. Soroko-Novickiy, B. Stechkin, A. Tolstov, G. Voshni etc.

The indicator efficiency coefficient shows only the losses caused by the internal irreversibility of the working processes, and does not take into account the losses from the external irreversibility associated with the final temperature difference between the heat sources and the working fluid. This does not consider and does not reveal the essence of the process of converting the thermal form of energy into mechanical work, but only describes the nature of the change in the indicator efficiency. In this regard, it is impossible to assess the degree of perfection and the nature of the impact on the efficiency of the in-cylinder engine processes.

From the system approach point of view, the piston engine represents an energy - generating system, in which various irreversible processes occur-combustion, heat and mass transfer, thermomechanical, etc. In this connection, it is advisable to consider the thermal machine as an open macroscopic system consisting of interconnected elements, each of which implements one or another irreversible thermodynamic process. Then, according to the second law of thermodynamics, the change of entropy plays a leading role in the description of such systems, and the indicator of irreversibility of the systems thermodynamic processes is the entropy production. Therefore, in the thermodynamic analysis of the piston engine cycle, it is advisable to predict the minimum expected 
damage in the form of thermal and "entropy" pollution along with the assessment of its efficiency and specific operation.

The main purpose of the article is to develop a strategy for the system analysis of the use of heat in piston engines, taking into account the non-equilibrium of heat and mass transfer processes in the over-piston space.

\section{Materials and methods}

It is known that in the classical approach based on the first law of thermodynamics the distribution of heat released during the combustion of fuel in the engine into individual components is expressed by the equation of the external heat balance and has the form [1]:

$$
Q_{e}=Q_{u}-\left(Q g+Q_{w}+Q_{l}+Q_{r}\right)
$$

where $Q_{u}$ - heat produced by fuel combustion;

$Q_{g}$ - heat carried away by exhaust gases (30-42\%);

$Q_{w}$ - heat removed by the cooling medium (14-28\%);

$Q_{l}$ - heat removed by lubricating oil (2-7 \%);

$Q_{r}$ - coefficient characterizing the unaccounted heat loss.

However, this approach in the description of energy balances does not allow to take into account the disparity of the converted part of the different energy types to the work, and makes it impossible to determine the location and causes of energy losses. This in its turn prevents to properly make their quantitative and qualitative assessment and to find ways to reduce energy losses [2]. In this regard, there is a need to study the working processes of piston engines to use both the first and second laws of thermodynamics and the basic principles of non-equilibrium thermodynamics. This approach allows us to take into account not only the quantity, but also the quality of energy.

In recent years, the thermodynamics of irreversible processes, which deals with nonequilibrium processes in time, has been widely developed. Modern thermodynamics of nonequilibrium processes was established in 50-70 of the last century and is associated with the names of L. Onsager, I. Prigogine, Dzh. Kayzer, S. De Grot, R. Haase and other scientists [3-7]. Thermodynamics of nonequilibrium processes allows us to study the process of entropy growth in more detail than classical thermodynamics and to calculate the amount of entropy formed in a unit volume per unit time due to the deviation of the system from the thermodynamic equilibrium.

At the same time, all thermodynamic processes occurring in the internal combustion engine (ICE), as in an open thermodynamic system, are not only irreversible, but also internally nonequilibrium and the duration of the processes is finite. So, this problem is best solved on the basis of the principles of thermodynamics at finite time[8-17]. Then there is a need to organize irreversible in-cylinder and heat and mass transfer processes in ICE systems so as to minimize energy dissipation in these processes at a given intensity of their flow. The search for the optimum target functions-minimizing the dissipation of thermal energy in non-equilibrium processes can be carried out among the obviously irreversible thermodynamic processes of piston engine systems with known heat transfer coefficients, specified durations and the amount of heat withdrawn (supplied) and as a result predict the maximum possible values of fuel and economic indicators of ICE.

To reveal the mechanism of energy losses in nonequilibrium thermodynamic processes occurring in piston engines, we can consider an expression for determining the amount of heat that is supplied to the open thermodynamic system at an infinitely small change of state [7]. The equation looks like:

$$
d_{e} Q=d U+p d V-d W_{d i s}-\sum_{k} H_{k} d n_{k},
$$

where $U, V, p$-internal energy, volume, system pressure;

$W_{\text {dis }}$ - work related to dissipative effects;

$H_{k}$ - partial molar enthalpy of the $k$-th component;

$d n_{k}$-increment of the number of moles of the $k$-th component of the working substance.

The processes occurring in the engine cylinder are due to the simultaneous change in the composition of the working fluid both due to heat and mass transfer $\left(d_{e} n_{k}\right)$, and due to chemical 
reactions $\left(d_{e} n_{k}\right)$. Therefore, an infinitesimal increment of the molar concentration of the components of the working body $n_{k}$ can be expressed as follows:

$$
d n_{k}=d_{e} n_{k}+d_{r} n_{k}=d_{e} n_{k}+\sum_{r} v_{k r} d \xi_{r},
$$

where $v_{k r}$-stoichiometric coefficient of the substance in the chemical reaction $r$ (positive for the formation of the substance and negative for its consumption as a result of the reaction); $\xi_{r}$ - degree of reaction completeness $r$.

Also, let us keep in mind that

$$
A_{r}=-\sum_{k=1}^{N} v_{k r} \mu_{k} \text { and } \mu_{k}=h_{k}-T S_{k}
$$

where $A_{r}$ - affinity of a chemical reaction $r$;

$S_{k}, \mu_{k}$ - partial molar entropy and chemical potential of the $k$-th component.

From formulas (2), (3), taking into account the relations (4), we can get the following expression:

$$
T d S=d_{e} Q+T \sum_{k} S_{k} d_{e} n_{k}+d W_{d i s}+\sum_{r} A_{r} d \xi_{r} .
$$

In this equation, the first and second terms of the right side - to heat and mass transfer with the external environment, the third term - to dissipative effects, the fourth term - to chemical reactions within the phase.

From equation (5) it follows that the amount of heat involved in an elementary, infinitely small, irreversible thermodynamic process is determined by the expression:

$$
\begin{aligned}
& d_{e} Q=T d S-T \sum_{k} S_{k} d_{e} n_{k}-d W-\sum_{r} A_{r} d \xi_{r}=T d S-d Q_{d i s s}= \\
& T d S-d_{i} Q=d Q-d_{i} Q,
\end{aligned}
$$

where $d Q$ - amount of heat involved in the elementary thermodynamic process;

$d_{e} Q$ - amount of heat supplied to the thermodynamic system from the heat source;

$d Q_{\text {diss }}$ - dissipated energy;

$d_{i} Q$ - uncompensated heat, depending on the degree of internal irreversibility thermodynamic processes, $d_{i} Q \geq 0$.

Thus, the amount of heat supplied to the differential volume in an elementary infinitesimal irreversible thermodynamic process from external sources $d_{e} Q$ :

$$
d_{e} Q=d Q-d_{i} Q .
$$

Thus, heat involved in any thermodynamic process of piston engines consists of two components: the first can have any sign depending on the direction of the heat flow, while the second cannot be negative.

Dividing both parts of the expression in formula (7) by the absolute temperature we obtain:

$$
d S=\frac{d_{e} Q}{T}+\frac{d_{i} Q}{T}=d_{e} S+d_{i} S .
$$

\section{Results and discussion}

For the study of heat in piston engines, the authors developed the equations $(9,10,12,14,15,17$, 20 ), allowing to solve the problem of thermodynamic process optimization in the system.

It follows from the above that the differential equation of the heat balance taking into account the total heat dissipation in the piston engine systems can be written as:

$$
d Q_{e}=d_{e} Q_{u}-\left(d e Q_{g}+d_{e} Q_{w}+d_{e} Q_{l}+\sum_{n} d_{i} Q_{n}+d Q_{r}^{\prime}\right) .
$$


Taking into account the expression (8), equation (9) can be transformed by changing the entropy to the form:

$$
d_{e} Q=d_{e} Q_{u}-T_{g}\left(d_{e} S_{g}+d_{i} S_{g}\right)-T_{w}\left(d_{e} S_{w}+d_{i} S_{w}\right)-T_{l}\left(d_{e} S_{l}+d_{i} S_{l}\right)-\sum d Q_{r},
$$

where $d_{e} S_{g}, d_{e} S_{w}, d_{e} S_{l}$ - reversible part of entropy change due to external heat exchange in exhaust, cooling and lubrication systems;

$d_{i} S_{g}, d_{i} S_{w}, d_{i} S_{l}$ - irreversible part of the entropy change due to the presence of nonequilibrium processes in the system of a piston engine.

This equation is the main one for the analysis of the heat use in the over-piston space.

According to the expression (8), the rate of growth of the total entropy in any engine system can be expressed as follows:

$$
d S / d t=d_{e} S / d t+d_{i} S / d t=-\int J_{s} d \Sigma+\int \sigma_{s} d V,
$$

where $J_{s}-$ full flow of entropy through the surface of the $\Sigma$ system;

$\sigma_{s}-$ local rate of entropy.

It follows from equation (10) that each $k$-th component of the heat balance can be represented as:

$$
Q_{k}=\int_{t} \int_{V} \rho_{k} T_{k} \frac{d S_{k}}{d t} d V_{k} d t_{k}=\int_{t} \int_{V} \rho_{k n} T_{k n} \sum_{n}\left(\frac{d_{e} S_{k n}}{d t}+\frac{d_{i} S_{k n}}{d t}\right) d V_{k n} d t,
$$

where $d_{e} S_{k n} / d t$ - rate of change of the irreversible part of the specific entropy in the $n$-th elementary thermodynamic process in the $k$-th system of the internal combustion engine.

$Q_{m}$ heat released in the combustion process is directly proportional to the amount of the burned fuel and depends on many factors affecting the perfection of the processes of fuel mixture formation and combustion. Therefore, due to the imperfection of the combustion process and under-heating of the fuel heat which stood out for the cycle is expressed for the calculation of the shares of the lowest combustion heat $H_{u}$ cyclic fuel dose:

$$
Q_{u}=H_{u} g_{z},
$$

where $\chi$-coefficient of heat release, taking into account the loss of part of the calorific value of the fuel due to incomplete combustion (lack of air, imperfect combustion);

$g_{z}$ - cyclic fuel supply;

$H_{u}$ - low heat of combustion.

The amount of heat lost from imperfection of the processes of mixture formation and combustion of fuel in the combustion chamber is equal $Q_{l}=(1-\chi) H_{u} g_{z}$. Then the uncompensated heat due to incomplete combustion of fuel can be written as:

$$
Q_{l}=(1-\chi) H_{u} g_{z}=T \Delta_{i} S_{C},
$$

where $\Delta_{\mathrm{i}} S_{c}$-total entropy production resulting from irreversible in-cylinder processes.

From the condition of additivity of entropy the total change of entropy in the energy-technological processes of the engine is the algebraic sum of the changes of entropy in the above-piston space and each of its systems, and has the form [18]:

$$
\sigma_{\Sigma}=\sum_{i} \Delta_{i} S / \tau=\left(\Delta_{i} S_{C}+\Delta_{i} S_{\lambda}+\Delta_{i} S_{w}+\Delta_{i} S_{g}+\Delta_{i} S_{l}\right) / \tau,
$$

where $\Delta_{\mathrm{i}} S_{\lambda}$ - total entropy production due to heat transfer and thermal conductivity of the cylinder wall;

$\Delta_{\mathrm{i}} S_{w}$ - total entropy production due to irreversible processes in the cooling system; $\Delta_{\mathrm{i}} S_{g}$ - total entropy production resulting from irreversible processes in the air supply system; 
$\Delta_{\mathrm{i}} S_{l}-$ total entropy production resulting from irreversible processes in the lubrication system.

The main task in assessing the efficiency of various processes is to study the process of converting the thermal form of energy into mechanical work in order to minimize energy losses in the engine systems. The solution of this problem allows us to determine the sources of irreversibility in the process. The essence of this problem is to establish the relationship between the increase in entropy in the thermodynamic system and the various irreversible thermodynamic processes occurring in it.

First of all, the balance equations for energy, mass and entropy are initially compiled. Further, from the equations of balance the functional relationship between a particular indicator of the efficiency of the thermodynamic process in the system of the internal combustion engine and dissipation $\sigma_{s}$ is established. Typically, the indicators of efficiency of the process deteriorate monotonically with the increase of dissipative losses and reach the maximum in a reversible process.

The last, the most difficult step is to solve the problem of such organization of the heat and mass transfer process in the ICE system, for which the heat dissipation will be minimal at given intensities of heat and mass flows. In general, the condition of minimum heat dissipation in irreversible processes can be represented as:

$$
\sigma_{\Sigma}=\frac{d_{i} S}{d t}=\frac{1}{t} \int_{0}^{t} \sum_{j=1}^{n} J_{j}\left(u_{1}, u_{2}\right) \cdot X_{j}\left(u_{1}, u_{2}\right) d t \rightarrow \min ,
$$

where $J_{j}, X_{j}$-thermodynamic flows and driving forces of irreversible processes;

$u_{i j}$ - process parameter.

The task of optimizing the thermodynamic process in the system is to select the temperatures, pressures and chemical potentials of interacting subsystems to achieve the minimum of entropy production at a given flow intensity. When optimizing thermodynamic parameters of in-cylinder processes, the degree of influence of the fuel combustion process parameters on them should also be taken into account [19].

The minimum value of the expression (14) characterizes the maximum energy efficiency of the piston engine, in which the irreversible loss of thermal energy will be the least. Consequently, the condition of minimal dissipation is reduced to the minimization of entropy production, which occurs due to the nonequilibrium of the above processes. Knowing the minimum value of the total entropy $\Sigma \Delta S_{i}(t)$, we can find the indicator power of the piston engine:

$$
N_{\max i}=\frac{1}{\tau} \iint_{t} \sum_{i=1}^{m} q_{i} d V d t \rightarrow \max ,
$$

where $\tau$-duration of the process of supply (removal) of heat in thermodynamic processes in the over-piston space.

The equation (17) follows from equations (12) and (13) as follows:

$$
N_{\max }=\frac{1}{\tau} \iint\left(d_{e} Q_{u}-d Q_{k}\right) d \tau=\frac{1}{\tau} \iint\left(\chi H_{u} d q_{z}-\sum_{k} d Q_{k}\right) d t=\frac{1}{\tau} \iint_{V} \sum_{i=1}^{m} q_{i} \cdot d V \cdot d \tau,
$$

where $q_{i}$ - relative speed of heat dissipation.

The equation (20) follows from the known formula of the indicator efficiency of the engine

$$
\eta_{i}=1-\frac{Q_{2 r}}{Q_{1}}=1-\frac{Q_{k}+Q_{d i s}^{\prime}}{Q_{1}}=1-\frac{\iint_{V} \rho_{k n} T_{k n} \sum_{n}\left(\frac{d_{e} S_{k n}}{d t}+\frac{d_{i} S_{k n}}{d t}\right) d V_{k n} d t+Q_{d i s}^{\prime}}{Q_{1}}=
$$




$$
=1-\frac{Q_{2 e}+Q_{d i s}^{\prime}+\sum_{n} \iint_{t} \rho_{V k} T_{k n} \sum_{n} \frac{d_{i} S_{k n}}{d t} V_{k n} d t}{Q_{1}},
$$

where $\rho_{k n}$ - density $k$ working body in $n$-th process;

$Q_{1}$ - amount of heat supplied from the hot source to the working body;

$Q_{2 e}$ - amount of heat taken away in reversible thermodynamic processes;

$Q_{d i s}$ - unaccounted dissipative heat losses in the piston engine.

So, the value of the indicator efficiency, taking into account the irreversibility of thermodynamic processes occurring in the internal combustion engine, can be determined by the formula:

$$
\eta_{i}=1-\frac{Q_{2 e}+Q_{d i s}^{\prime}+\sum_{n} \iint_{t} \rho_{V} T_{k n} \sum_{n} \frac{d_{i} S_{k n}}{d t} V_{k n} d t}{Q_{1}} .
$$

This equation allows us to estimate the degree of the supplied heat use.

\section{Conclusions}

The real thermodynamic processes occurring in piston engines are irreversible. They proceed with a finite intensity of heat transfer, and their duration is finite. Usually, the results of evaluating the effectiveness of real in-cylinder processes are overestimated, which does not allow to objectively judge the maximum engine power.

This article presents a method of thermodynamic analysis of the efficiency of the transfer processes and conversion of the energy thermal form in the engine cylinder, taking into account their nonequilibrium. The proposed entropy approach using the principles of nonequilibrium thermodynamics and thermal dissipation makes it possible to more realistically assess the degree of heat use.

Further direction of the research is practical calculation of the engine performance indicators on the developed model.

As a result of the research the following conclusions are obtained:

1. The use of the entropy approach based on the concept of dissipative function of the physicochemical subsystem allows to investigate the maximum capabilities of the piston engine.

2. Energy losses of heat and mass transfer processes in internal combustion engine systems are estimated by the nature of entropy growth.

3. The conditions of minimal dissipation include functions that determine the laws of heat and mass transfer in thermodynamic systems, the rate of supply and removal of heat in the engine cylinder, etc.

4. As the criterion in this problem is the minimum irreversible loss of the function linking the variables of the contact systems with the flow of the exchange, it will determine the properties of the optimal control.

\section{References}

[1] Sharoglazov B. A., Shishkov V. V. Piston engines: theory, modeling and analysis of workflows. Chelyabinsk: UrSU, 2011. 525p.

[2] Gurov K. P. Phenomenological thermodynamics of irreversible processes. Moskva: Nauka, 1978. $128 \mathrm{p}$.

[3] Ge Groot S., Mazur P. Nonequilibrium thermodynamics - Moskva: Mir, 1964. 456 p.

[4] Kaiser G.. Statistical thermodynamics of nonequilibrium processes.Moskva:Mir, 1990, 608 p.

[5] Prigozhin I., Kondepudi D. Modern thermodynamics. From heat engines to dissipative structuresMoskva: Mir. 2002. - $461 \mathrm{p}$.

[6] Onsager L. Reciprocal relations in irreversible thermodynamics// Physical Review, 1931, No. 37, pp. 405-426. 
[7] Haase R. Thermodynamics of irreversible processes. - Moskva: Mir, 1967, 544 p.

[8] Mironov V. A. and others Mathematical methods of thermodynamics at finite time. - Moskva: Chemistry, 2000, 379 p.

[9] Vamshikrishna A. Reddy, Sharathkumar T., Tharunkumar D.K., Dinesh B., Saisantosh Y.V.S. Energy and Exergy Analysis of I.C. Engines The International Journal Of Engineering And Science V 3(5) 2014, pp. 7-26.

[10] Ahmed F., Rashad M. Energy and Exergy Analysis of Internal Combustion Engine with Injection of Hydrogen into the Intake Manifold International Journal of Energy Engineering V5(6), 2015, pp. 163-170.

[11]Bourhis G., Leduc P., Bourhis G., Leduc P. Energy and Exergy Balances for Modern Diesel and Gasoline Engines Oil \& Gas Science and Technology - Rev. IFP, V65(1) 2010, pp. 39-46.

[12] Jorge J., G. Martins, Bernardo S., Ribeiro, Ion V. Ion. Thermodynamic analysis of Spark Ignition engines using the Entropy Generation Minimisation method. International Journal of Exergy, Vol. 6, No. 1, 2009, pp. 93-110.

[13] Jelić M.,, Ninić N. Analysis of Internal Combustion Engine Thermodynamic Using the Second Law of Thermodynamics, // Strojarstvo 50 (2), 2008, pp. 85-94.

[14] Ashok Reddy K. A Crtical Review of Entropy Generation in Internal Combustion Engine // Dept of Mechanical Engineering, MLR Institute of Technology , Dundegal (V), Hyderabad-43.Volume 4, Issue 3, March 2016, pp. 1-4.

[15] Kamboj S.K., Karimi M.N. Exergy Analysis of Internal Combustion Engine Based Cooling Cycle // Int. J. of Thermal \& Environmental Engineering Vol. 16, No. 2, 2018, pp. 113-118.

[16] Ribeiro B., Martins J., Nunes A. Generation of Entropy in Spark Ignition Engines // Int. J. of Thermodynamics ISSN 1301-9724 Vol. 10 (No. 2), 2007, pp. 53-60.

[17] Salamon P. Physics versus engineering of finite-time thermodynamic models and optimizations // Thermodynamic optimization of complex energy systems. - Dordrecht: Kluwer, 1999, pp. 421-424.

[18]Zainetdinov R. A. Dynamics of reciprocating engines. - SPb: SPbSAU, 2018, 272 p.

[19]Zainetdinov R. A. Features of the process of transfer and transformation of heat-ing energy in the combustion chamber of the piston engine//Proceedings of Saint-Petersburg State Agrarian University, 2018, No 52, pp. 214-220. 